\title{
Dynamical phase transition to localized states in the two-dimensional random walk conditioned on partial currents
}

\author{
Ricardo Gutiérrez $\oplus^{1}$ and Carlos Pérez-Espigares $\circledast^{2,3}$ \\ ${ }^{1}$ Complex Systems Interdisciplinary Group (GISC), Department of Mathematics, Universidad Carlos III de Madrid, \\ Leganés 28911, Madrid, Spain \\ ${ }^{2}$ Departamento de Electromagnetismo y Física de la Materia, Universidad de Granada, Granada 18071, Spain \\ ${ }^{3}$ Institute Carlos I for Theoretical and Computational Physics, Universidad de Granada, Granada 18071, Spain
}

(Received 2 September 2021; accepted 12 October 2021; published 27 October 2021)

\begin{abstract}
The study of dynamical large deviations allows for a characterization of stationary states of lattice gas models out of equilibrium conditioned on averages of dynamical observables. The application of this framework to the two-dimensional random walk conditioned on partial currents reveals the existence of a dynamical phase transition between delocalized band dynamics and localized vortex dynamics. We present a numerical microscopic characterization of the phases involved and provide analytical insight based on the macroscopic fluctuation theory. A spectral analysis of the microscopic generator shows that the continuous phase transition is accompanied by spontaneous $\mathbb{Z}_{2}$-symmetry breaking whereby the stationary solution loses the reflection symmetry of the generator. Dynamical phase transitions similar to this one, which do not rely on exclusion effects or interactions, are likely to be observed in more complex nonequilibrium physics models.
\end{abstract}

DOI: 10.1103/PhysRevE.104.044134

\section{INTRODUCTION}

The study of large fluctuations of dynamical observables in lattice gas models has greatly improved our understanding of nonequilibrium statistical mechanics, as it has shed light on rare events in transport phenomena [1-3], has unveiled fluctuation relations of wide applicability [4-9], and has clarified the origin of many-body effects in classical $[10,11]$ and quantum systems [12-14]. These microscopic analyses have been complemented with approaches based on the macroscopic fluctuation theory, where similar phenomena are studied at a coarse-grained level [15]. Both frameworks rely on statistical ensembles of trajectories [16,17], for which large deviation functions play an analogous role to that of thermodynamic potentials in equilibrium statistical mechanics [18].

Dynamical phase transitions (DPTs), characterized by sudden changes in the structure of trajectories that are reflected in the statistics of dynamical observables, are among the most interesting phenomena that have been unveiled by these approaches. DPTs manifest themselves as nonanalyticities in the large deviation functions and occur not only in systems driven far from equilibrium but also in equilibrium settings, even in situations where the statistics of (static) configurations is trivial [11]. In recent years, the study of DPTs has been enriched by the application of the generalized Doob transform, which provides the subset of trajectories sustaining a given rare fluctuation [19-23], allowing for characterization of dynamical phases.

The study of DPTs is frequently accomplished in onedimensional systems [24-38], where the computation of large deviations for large systems is analytically tractable. Analyses of higher dimensional settings are challenging, since they rely on variational procedures and diagonalization problems whose complexity increases with the spatial dimension. Yet the statistics of total currents (quantifying the net number of particles crossing a section that spans the whole system) or dynamical activities (counting configuration changes in a trajectory) have been studied in two dimensions, where a myriad of phenomena, including DPTs, have been reported [39-44]. In the case of a two-dimensional random walk on a lattice, the Doob-transformed dynamics that sustains a given fluctuation of the total current amounts to the inclusion of a uniform driving field. This implies that large fluctuations are achieved by flat, i.e., lacking spatial structure, density, and current fields, giving rise to current distributions that are trivially Gaussian. The fluctuations of so-called partial currents, i.e., currents of particles that move across some "wall" or "slit" that does not span the whole system, are strikingly different, however, as we shall show.

In this paper, we consider the statistics of partial currents across a finite slit of random walks on a square lattice, as well as its continuum hydrodynamic limit. We find that, despite the simplicity of the model, there is a DPT underpinning rare fluctuations of such partial currents, which is continuous and characterized by a spontaneous breaking of the $\mathbb{Z}_{2}$ reflection symmetry in the direction of the slit. While small fluctuations are characterized by the formation of bands, rare fluctuations condense into localized vortices. This highlights how large fluctuations of partial currents, which are more relevant than total currents in experimental settings, are created by nontrivial emerging structures associated with a DPT. Given the simplicity and generality of the model, similar DPTs are likely to be found in other systems with exclusion effects and interactions, and might shed light on intriguing results previously reported for the two-dimensional simple exclusion process [45]. Regarding the observability of the DPT under consideration, it seems realistic that it may be observed in 
experiments such as those devised to study global current fluctuations in diffusive systems $[46,47]$. In fact, experimental work designed to measure the statistics of other local observables has been successfully carried out in different settings; see, e.g., Refs. [48,49].

\section{MODEL}

We consider $N$ random walkers on a two-dimensional square lattice comprising $L \times L$ sites with periodic boundary conditions in the presence of a driving field $\mathbf{E}=$ $\left(E_{x}, E_{y}\right)$, which induces asymmetric jump rates between neighboring sites. The stochastic dynamics of each particle is governed by the master equation $\partial_{t} p(n, t)=-p(n, t)+$ $\sum_{m \in v_{n}} \Gamma_{n \leftarrow m} p(m, t)$, where $p(n, t)$ is the probability of occupation of site $n \in\left\{1,2, \ldots, L^{2}\right\}$ at time $t$, and $v_{n}$ contains its (four) nearest neighbors. Written in operatorial form, $d \mathbf{p}(t) / d t=\mathbb{W} \mathbf{p}(t)$, with the column vector $\mathbf{p}(t)=$ $\left(p(1, t), p(2, t), \ldots, p\left(L^{2}, t\right)\right)^{T}$ (T denotes transposition), and the entries of the generator $\mathbb{W}_{n n^{\prime}}=-\delta_{n n^{\prime}}+\Gamma_{n \leftarrow n^{\prime}}\left(\Gamma_{n \leftarrow n^{\prime}}=0\right.$ if $n^{\prime} \notin v_{n}$ ). The transition rates are

$$
\Gamma_{n \leftarrow m}=e^{\frac{\mathbf{E} \cdot\left(\mathbf{r}_{n}-\mathbf{r}_{m}\right)}{L}} /\left(e^{\frac{E_{X}}{L}}+e^{-\frac{E_{x}}{L}}+e^{\frac{E_{y}}{L}}+e^{-\frac{E_{y}}{L}}\right),
$$

so that $\sum_{n} \Gamma_{n \leftarrow m}=1$, where $\mathbf{r}_{n}$ is the position vector of site $n$ taking the lattice constant as spatial unit. The observable of interest is the partial current $J$ across a vertical slit of length $h L(0<h<1)$ placed in the center of the lattice, which we take as the origin of (Cartesian) coordinates. $J$ quantifies the number of particles that traverse the slit in the rightward direction minus those that do in the leftward direction per unit of time.

\section{MICROSCOPIC LARGE-DEVIATION METHODS}

The current $J$ follows a probability distribution that adopts a large deviation form for long times, $P(J) \sim e^{-t \varphi(J)}$, with rate function $\varphi(J)$, which is non-negative and equal to zero only for the average current $J=\langle J\rangle$ [16]. For simplicity, in the following we will consider a horizontal field $\mathbf{E}=$ $(E, 0)$ giving rise [cf. (1)] to an average partial current $\langle J\rangle=$ $\rho_{0} h L \tanh [E / 2 L]$, which in the limit of large $L$ becomes $\langle J\rangle=$ $\rho_{0} E h / 2$. To investigate the fluctuations of $J$, one would like to find the rate function $\varphi(J)$, but this is in general a difficult task, as we are dealing with events that are exponentially unlikely in time. Instead, we bias the probability distribution with a parameter $s$, obtaining a new distribution $P_{s}(J)=e^{-s t J} P(J) / Z_{s}$, where the dynamical partition function $Z_{s}$ acquires for long times the following large deviation form,

$$
Z_{s}=\int d J e^{-s t J} P(J) \sim e^{t \theta(s)} .
$$

The scaled cumulant-generating function (SCGF) $\theta(s)$ is related to the rate function by a Legendre-Fenchel transform, $\theta(s)=-\min _{J}[s J+\varphi(J)][16]$. By choosing the appropriate value of $s$, we find the fluctuation of interest, $J=$ $\langle J\rangle_{s}$ (where $\langle\cdot\rangle_{s}$ is the average over $P_{s}(J)$ ), which in turn corresponds to (minus) the first derivative of the SCGF, $\langle J\rangle_{s}=-\theta^{\prime}(s)$. In general, the $p$ th derivative of the SCGF yields the partial-current cumulant of the corresponding order $\left\langle\left\langle J^{p}\right\rangle\right\rangle_{s}: \lim _{t \rightarrow \infty} t^{p-1}\left\langle\left\langle J^{p}\right\rangle\right\rangle_{s}=(-1)^{p} \frac{d^{p} \theta(s)}{d s^{p}}[11]$.
The SCGF can be obtained as the largest eigenvalue of the so-called tilted generator $W^{s}$, whose entries are [16]

$$
\mathbb{W}_{n n^{\prime}}^{s}= \begin{cases}e^{-s} \mathbb{W}_{n n^{\prime}} & n \in n_{r} \text { and } n^{\prime} \in n_{l}, \\ e^{s} \mathbb{W}_{n n^{\prime}} & n \in n_{l} \text { and } n^{\prime} \in n_{r}, \\ \mathbb{W}_{n n^{\prime}} & \text { otherwise, }\end{cases}
$$

where $n_{r}$ contains all sites that are immediately to the right of the slit, and $n_{l}$ those that are immediately to the left. Notice that in Eq. (3), $s=0$ corresponds to the original dynamics, while $s<0$ enhances current fluctuations larger than the average, and the opposite occurs for $s>0$. Since $W^{s}$ is not symmetric, it has different left and right eigenvectors, which satisfy $\mathbf{l}_{s, i}^{T} \mathbb{W}^{s}=\lambda_{i}(s) \mathbf{l}_{s, i}^{T}$ and $\mathbb{W}^{s} \mathbf{r}_{s, i}=\lambda_{i}(s) \mathbf{r}_{s, i}$, respectively, where $i=0,1, \ldots, L^{2}-1$. The eigenvalues $\lambda_{i}(s)$ are ordered in decreasing magnitude of the real part, and $\lambda_{0}(s)=\theta(s)$. While it might appear natural that the tilted dynamics generated by (3) must be a biased random walk, it turns out that such dynamics is not physical, as $\mathbb{W}^{s}$ does not conserve probability, $\sum_{n} \mathbb{W}_{n n^{\prime}}^{s} \neq 0$. To recover the physical dynamics sustaining the fluctuation associated with a given $s$ a transformation is needed, namely the generalized Doob transform [19-23],

$$
\mathbb{W}_{\text {Doob }}^{s}=L_{s} \mathbb{W}^{s} L_{s}^{-1}-\theta(s) \mathbb{1} .
$$

Here $L_{s}$ is the diagonal matrix whose entries are the components of the leading left eigenvector, $\mathbf{l}_{s, 0}$, and $\mathbb{1}$ is the $L \times L$ identity matrix. The Doob generator $W_{\text {Doob }}^{s}$ is a proper stochastic (probability conserving) generator, $\sum_{n} \mathbb{W}_{\text {Doob }, n n^{\prime}}^{s}=$ 0 . Its eigenvalues are $\lambda_{i}^{D}(s)=\lambda_{i}(s)-\theta(s)$, with left eigenvectors $\mathbf{l}_{s, i}^{D}=L_{s}^{-1} \mathbf{l}_{s, i}$ and right eigenvectors $\mathbf{r}_{s, i}^{D}=L_{s} \mathbf{r}_{s, i}$. The largest (zero) eigenvalue is associated with the stationary state $\mathbf{p}_{\text {stat }}^{s}=\mathbf{r}_{s, 0}^{D}, W_{\text {Doob }}^{s} \mathbf{p}_{\text {stat }}^{s}=0$ [50]. The Doob generator (4) provides the stochastic process whose long-time statistics of $J$ is given by $P_{S}(J)$.

For one random walk sustaining the fluctuation $J$, the density and current fields are thus given by $p_{\text {stat }}^{s}(n)$ (which are the entries of $\left.\mathbf{p}_{\text {stat }}^{s}\right)$ and $\mathbf{j}^{s}(n)=\left(j_{x}^{s}(n), j_{y}^{s}(n)\right)$. The latter satisfies $\sum_{n \in n_{l}} j_{x}^{s}(n)=J$ and can be obtained from the difference between the probability fluxes of two adjacent sites, $j_{\alpha}^{s}(n)=p_{\text {stat }}^{s}(n) W_{\text {Doob }, m n}^{s}-p_{\text {stat }}^{s}(m) W_{\text {Doob }, n m}^{s}$, where $m$ is the right (upper) neighbor of $n$ for $\alpha=x(\alpha=y)$. For $N$ particles, the density field, namely the average number of particles per site, is just $\rho^{s}(n)=N p_{\text {stat }}^{s}(n)$, and the current field is $\emptyset^{s}(n)=$ $N \mathbf{j}^{s}(n)$. The SCGF must also be multiplied by $N$, as can be seen from the stochastic independence of different random walks, which yields $Z_{s}^{N}$ as the dynamical partition function, $Z_{s}$ being the one-particle partition function (2).

\section{MICROSCOPIC ANALYSIS OF DYNAMICAL REGIMES}

In Figs. 1(a) and 1(b), we provide a first glimpse of the fluctuations of the partial current $J$ in a system of linear size $L=32$ with a slit of relative length $h=1 / 2$ under a field of strength $E=5$. In the main panel of Fig. 1(a), we show the (rescaled) SCGF $L^{2} \theta(s)$ and the corresponding current fluctuation, $L^{2}\langle J\rangle_{s}$ for different values of the biasing field $s$. Since $N=\rho_{0} L^{2}$, where $\rho_{0}$ is the total density, we represent $\theta(s)$ and derived quantities multiplied by $L^{2}$, which corresponds to fixing the density to $\rho_{0}=1$, and will be useful in the comparison of results for different size $L$ later. When a 
(a)

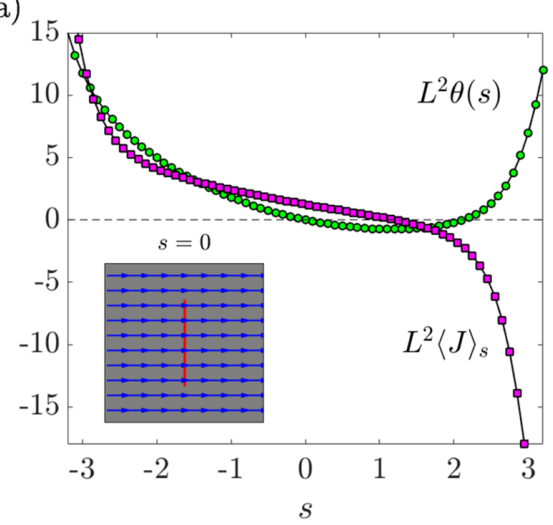

(b)

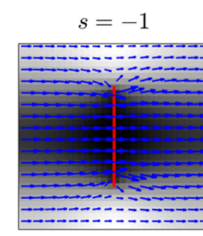
$s=1$

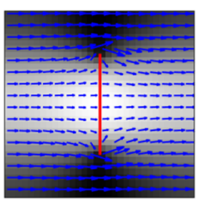

$s=-3$

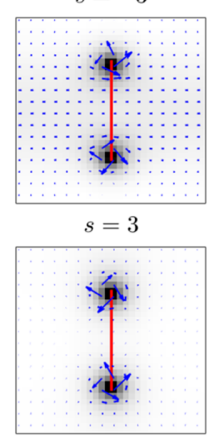

(c)

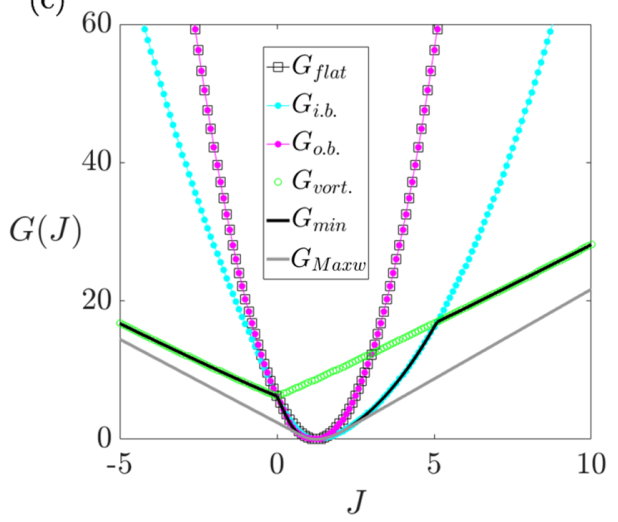

FIG. 1. Microscopic and macroscopic large-deviation analysis of the partial current statistics in the two-dimensional random walk. (a) (Main) Rescaled SCGF $L^{2} \theta(s)$ (green circles) and average partial current $L^{2}\langle J\rangle_{s}$ (magenta squares) for linear size $L=32$, relative slit length $h=1 / 2$ and field strength $E=5$. (Inset) Density field $\rho^{s}(n)$ (gray color map, darker color indicates a larger number of particles) and current field $\AA^{s}(n)$ (blue arrows) for $s=0$ (natural dynamics). The slit is highlighted in red. (b) Density field $\rho^{s}(n)$ and current field $\AA^{s}(n)$ for $s=-1$ (corresponding to $\left.L^{2}\langle J\rangle_{s}=2.42\right), s=-3\left(L^{2}\langle J\rangle_{s}=13.09\right), s=1\left(L^{2}\langle J\rangle_{s}=0.23\right)$, and $s=3\left(L^{2}\langle J\rangle_{s}=-20.33\right)$, with format as in the inset of panel (a). (c) Macroscopic fluctuation theory functional for the flat solution $G_{\text {flat }}(J)$, the inner-band solution $G_{\text {i.b. }}(J)$, the outer-band solution $G_{\text {o.b. }}(J)$, and the vortex solution $G_{\text {vort. }}(J)$. The minimum value among them corresponding to each $J$, denoted $G_{\min .}(J)$, is also shown, as well as its convex envelope $G_{\text {Maxw. }}(J)$. See text for definitions and the Appendices for details.

comparison is made for a fixed number of particles $N$ instead, we shall focus on one-particle quantities $(N=1)$, but then of course the density will vary as $\rho_{0}=1 / L^{2}$.

For $s=0$, the density and the current fields are uniform, see inset of Fig. 1(a), and the partial current is $L^{2}\langle J\rangle_{s=0}=$ $\langle J\rangle=1.25$, which corresponds to the expression given above for $\rho_{0}=1$. For $s>0, L^{2}\langle J\rangle_{s}$ decreases monotonically slowly up to $s \approx 2$, and then more rapidly, while a similar but opposite behavior is found for $s<0$. The density and current fields $\left\{\rho^{s}(n), \Phi_{D^{s}}(n)\right\}$ for $s \neq 0$ are displayed in Fig. 1(b).

For negative tiltings, $s<0$, the density becomes higher in the horizontal band crossing the slit, i.e., $y \in(-h L / 2, h L / 2)$, as illustrated for $s=-1$. This regime, which will be referred to as the inner band, persists for a range of negative values of $s$, with a spatial decay for $|y|>h L / 2$ that becomes more abrupt as the absolute value of $s$ increases. The band is not perfectly uniform in the horizontal direction, however, as there is a tendency for particles to populate the edges of the slit and to move cyclically around them-an effective way to increase the partial current $J$-which is also enhanced as the absolute value of $s$ increases. Beyond a certain point, the band practically disappears, and the (large) current is almost completely sustained by vortices localized at the edges, as shown for $s=-3$. Such cyclic, localized behavior, which has recently been observed in random walks on general graphs [51] and the zero-range process on a diamond lattice [52], presents some similarities to the vortices of the two-dimensional simple exclusion process [45].

For positive and moderately large values of $s$, the relatively low $J$ is achieved by forming a band that avoids passing through the slit, the bulk of the density showing in the region where $|y|>h L / 2$, which we will call the outer band, and is illustrated for $s=1$. But also here there is a tendency of particles to populate the edges of the slit and move in circles (this time in the opposite direction, so the current decreases with the adopted sign convention). For sufficiently large $s$, as illustrated for $s=3$, the band again practically disappears, leading to a vortex dynamics of opposite vorticity to that observed for negative $s$. This phenomenology will be shown to be associated with a DPT to a localized state. But before addressing its nature, a macroscopic analysis will provide further insight into the dynamical regimes at play.

\section{MACROSCOPIC ANALYSIS OF DYNAMICAL REGIMES}

Following the macroscopic fluctuation theory, which studies fluctuations of dynamical observables at the macroscopic (coarse-grained) level [15], we consider the probability of any trajectory of duration $T$ given by the density and current fields $\{\rho(\mathbf{r}, t), \mathbf{j}(\mathbf{r}, t)\}_{0}^{T}$. For driven diffusive systems, such probability adopts a large-deviation form $P\left[\{\rho(\mathbf{r}, t), \mathbf{j}(\mathbf{r}, t)\}_{0}^{T}\right] \sim$ $e^{-L^{2} I[\rho, \mathrm{j}]}$, with the rate functional

$$
I[\rho, \mathbf{j}]=\int_{0}^{T} d t \int_{\Lambda} d \mathbf{r} \frac{[\mathbf{j}+D(\rho) \nabla \rho-\sigma(\rho) \mathbf{E}]^{2}}{2 \sigma(\rho)} .
$$

For a random walk, the diffusivity is $D(\rho)=1 / 4$ and the mobility $\sigma(\rho)=\rho / 2$ [53]. A diffusive rescaling of the microscopic variables, whereby time is rescaled by $1 / L^{2}$ and space by $1 / L$, so that the process takes place over $\Lambda=[-1 / 2,1 / 2] \times[-1 / 2,1 / 2]$, is applied. From Eq. (5), we can calculate by contraction the probability of any observable depending on the trajectory. Thus the probability of having a current $J$ through the slit reads $P(J) \sim$ $\exp \left\{-T L^{2} G(J)\right\}$, with $G(J)=\lim _{T \rightarrow \infty} \frac{1}{T} \min _{\{\rho, \mathbf{j}\}_{0}^{T}}^{*} I[\rho, \mathbf{j}]$. Here $*$ means that the minimization is subject to the constraints $J=T^{-1} \int_{0}^{T} d t \int_{-h / 2}^{h / 2} d y j_{x}(0, y ; t)$ and $\partial_{t} \rho=-\nabla \cdot \mathbf{j}$ (continuity equation). Notice that the probability is maximized $[G(J)$ is zero] when $\mathbf{j}(\mathbf{r}, t)$ equals the macroscopic average current $\mathbf{j}=-D(\rho) \nabla \rho+\sigma(\rho) \mathbf{E}$.

Since solving the two-dimensional spatiotemporal variational problem for density and current fields such as those displayed in Fig. 1(b) is a daunting task, we shall focus on 
a few idealized ansätze for $\{\rho(\mathbf{r}), \mathbf{j}(\mathbf{r})\}_{0}^{T}$. Both their time independence and their structural features are based on the previous microscopic results. Specifically, we consider four dynamical regimes, each one leading to a different form of $G(J)$ : (i) a flat solution (uniform density and current fields) $G_{\text {flat }}(J)$, (ii) an inner band solution (uniform density over the region of $\Lambda$ where $|y|<h / 2) G_{\text {i.b. }}(J)$, (iii) an outer band solution (uniform density over the region of $\Lambda$ where $|y|>h / 2$ ) $G_{\text {o.b. }}(J)$, and (iv) a vortex solution $G_{\text {vort. }}(J)$, all of which are discussed in the Appendices. Figure 1(c) shows $G_{\min .}(J)=$ $\min \left\{G_{\text {flat }}(J), G_{\text {i.b. }}(J), G_{\text {o.b. }}(J), G_{\text {vort. }}(J)\right\}$, which maximizes the probability at each $J$, yielding

$$
G_{\min .}(J)= \begin{cases}G_{\text {vort. }}(J) & J<0, \\ G_{\text {o.b. }}(J) & 0 \leqslant J<\langle J\rangle, \\ G_{\text {flat }}(J) & J=\langle J\rangle, \\ G_{\text {i.b. }}(J) & \langle J\rangle<J<\tilde{J}, \\ G_{\text {vort. }}(J) & \tilde{J} \leqslant J .\end{cases}
$$

While the flat solution maximizes the probability for $J=\langle J\rangle$, its Gaussian fluctuations around $\langle J\rangle$ are suppressed exponentially in time with respect to those of the bands: Immediately to the right of $\langle J\rangle$ the dominant regime is the inner band, while the outer band prevails for values of $J$ between zero and $\langle J\rangle$. For larger fluctuations, $G_{\text {vort. }}(J)$ dominates for $J<0-$ counterclockwise rotation in the upper edge and clockwise rotation in the lower edge of the slit-and also for $J>\tilde{J}-$ with vortices rotating in the opposite directions-where $\tilde{J}$ is to an extent dependent on the choice of a cutoff radius (see the Appendices).

While a Legendre-Fenchel transformation of $G_{\min }(J)$ yields the macroscopic SCGF of Fig. 2(b) (red discontinuous line; see the discussion below), an inverse Legendre-Fenchel transformation applied on such SCGF gives the convex envelope $G_{\text {Maxw. }}(J)$, which, unlike $G_{\text {min. }}(J)$ itself, is convex; see Fig. 1(c). This Maxwell construction highlights a coexistence between dynamical phases [16], namely, that of vortices and bands, specifically the outer band for $J<\langle J\rangle$ and the inner band for $J>\langle J\rangle$. Though this is broadly in agreement with the microscopic results of Fig. 1(b), it should not be taken as indicative of the existence of a first-order DPT, as $G_{\min .}(J)$ is restricted to a few idealized cases, and is thus an upper bound of the actual macroscopic functional: Such coexistence may well approximate dynamical regimes not fully describable in terms of the ansätze under consideration.

\section{MICROSCOPIC ANALYSIS OF THE DPT}

In order to elucidate the nature of the DPT, we again focus on the microscopic SCGF $\theta(s)$. In Fig. 2(a), we show the (rescaled) SCGF $L^{2} \theta(s)$ for different linear sizes ranging from $L=12$ to 200. A good collapse of $L^{2} \theta(s)$ curves is found for $|s| \lesssim 2$, which marks the onset of the DPT, as can be better appreciated in Fig. 2(b), which also includes the macroscopic SCGF discussed in the previous section (red discontinuous line). Such an agreement, however, does not exist for $|s|$ larger than critical, as $L^{2} \theta(s)$ then grows without bound as $L$ increases, yielding a rate function $\varphi(J)$ that becomes linear [qualitatively similar to $G_{\text {Maxw. }}(J)$ in Fig. 1(c)], and therefore
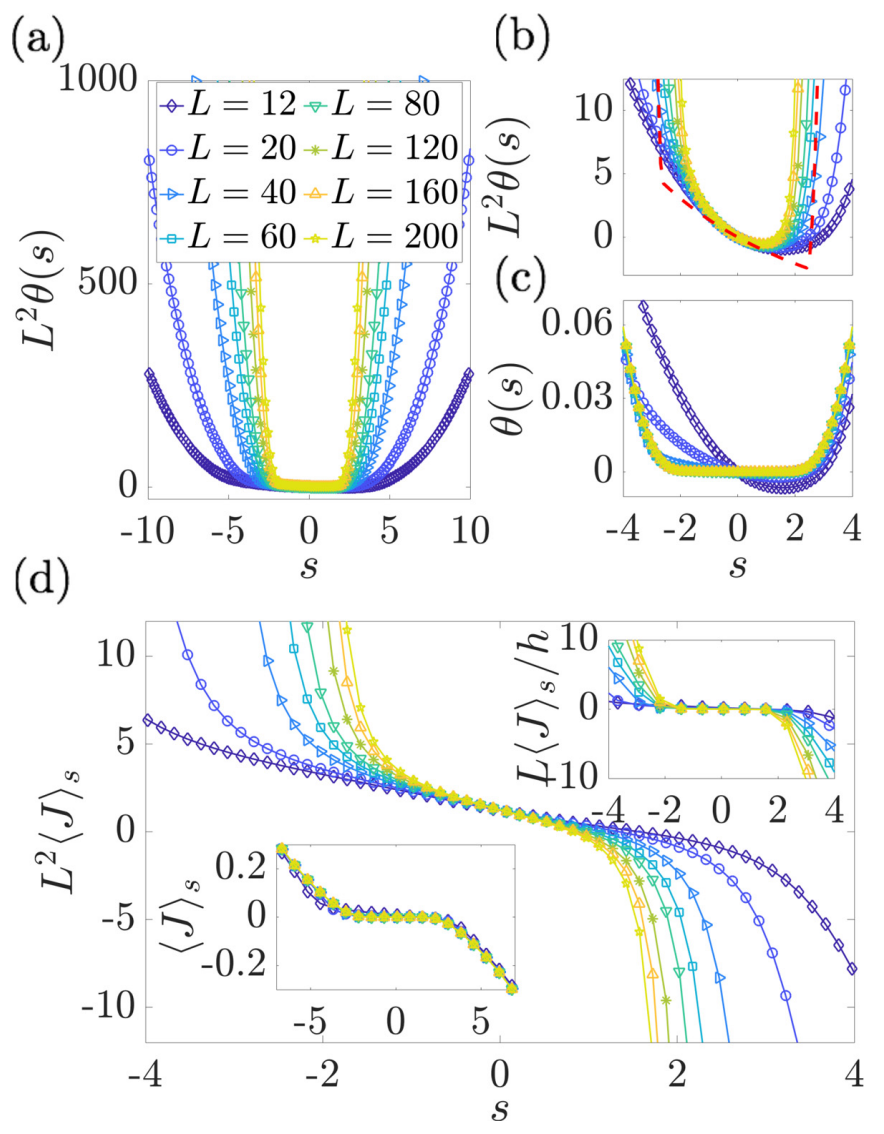

FIG. 2. Characterization of the DPT: SCGF and average current. (a) Density-rescaled SCGF $L^{2} \theta(s)$. (b) Same content as in panel (a) but enlarged around small values of $|s|$ and also including macroscopic SCGF (red discontinuous line). (c) SCGF $\theta(s)$. (d) Average current $\langle J\rangle_{s}$ multiplied by $L^{2}$ (main panel), by $L / h$ (top right inset) and without rescaling (lower inset). See text for justifications of rescalings. In all panels, the colors and symbols represent different system sizes $L$ according to the legend in panel (a).

an asymptotically exponential distribution $P(J)[16]$. Nevertheless, a good collapse is found beyond the critical points if the rescaling of $\theta(s)$ by $L^{2}$ is not performed (i.e., if the number of particles $N$, instead of the density $\rho_{0}$, is kept fixed), as shown in Fig. 2(c). Similar conclusions can be drawn from the average current $\langle J\rangle_{s}$, which is shown in rescaled form $L^{2}\langle J\rangle_{s}$ in the main panel of Fig. 2(d). After further normalization by the slit length $h L, L\langle J\rangle_{s} / h$, displayed in the upper inset, shows a good collapse below the critical points, but certainly not for larger absolute values of $s$. There the collapse is observed in the unrescaled form $\langle J\rangle_{s}$ (for fixed particle number $N$, without normalizing by the slit size), as shown in the lower inset.

We conclude that a collapse for different sizes $L$ occurs below the critical point for fixed density $\rho_{0}$, while on the other side of the transition it is the number of particles $N$ that must be kept constant. Viewed from the perspective of the idealized regimes of the previous section, the current associated with band dynamics is proportional to $\rho_{0}$, while that of localized vortices is proportional to the number of particles $N$.

The features of the DPT can be inferred from the spectral properties of the Doob generator $W_{\text {Doob }}^{s}$ $[34,38,54,55]$, in particular from the analysis of the 
(a)

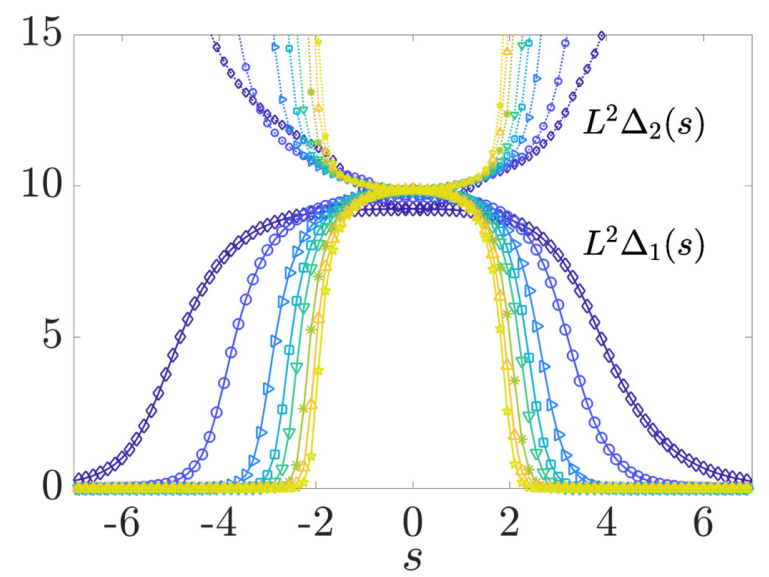

(b)

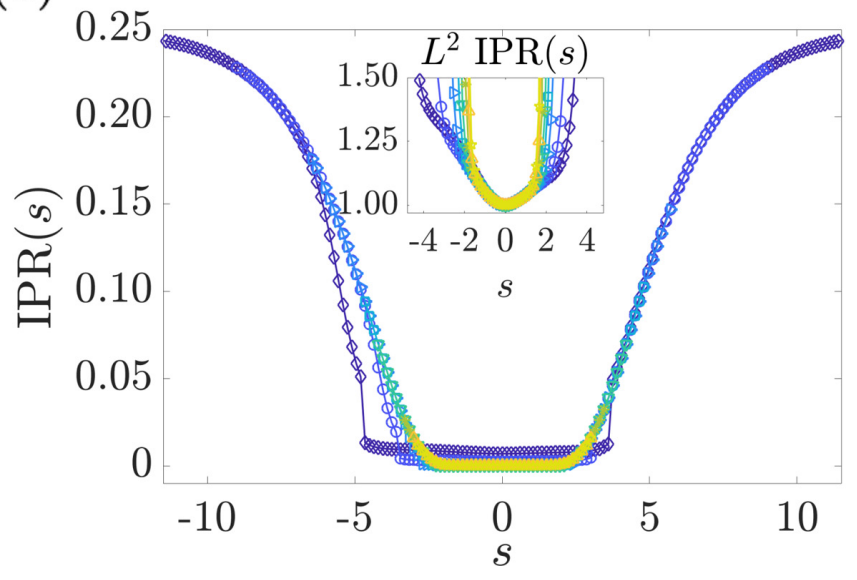

FIG. 3. Characterization of the DPT: spectral gaps and inverse participation ratio. (a) Rescaled spectral gaps $L^{2} \Delta_{1}(s)$ (continuous lines, large symbols) and $L^{2} \Delta_{2}(s)$ (dotted lines, small symbols); see text for definitions. (b) Inverse participation ratio, without (main panel) and with rescaling (inset). As $L$ increases, the range of $s$ explored decreases, due to convergence issues of the eigenvalue algorithm. In all panels, the colors and symbols represent different system sizes $L$ according to the legend in Fig. 2(a).

spectral gaps $\quad \Delta_{i}(s)=\theta(s)-\operatorname{Re}\left[\lambda_{i}(s)\right]=-\operatorname{Re}\left[\lambda_{i}^{D}(s)\right]$ (by definition $\Delta_{0}(s)=0$ ). The first two, $\Delta_{1}(s)$ (usually referred to as the spectral gap) and $\Delta_{2}(s)$, are shown in rescaled form (multiplied by $L^{2}$ so as to fix the density, $\left.\rho_{0}=1\right)$ in Fig. 3(a). While $L^{2} \Delta_{2}(s)$ only grows with $|s|$, we observe that the gap $L^{2} \Delta_{1}(s)$ closes at a certain value, which accumulates around the critical region at the onset of the DPT. As $\lambda_{1}^{D}(s)$ is real across the range of $s$ considered (unlike $\lambda_{2}^{D}(s)$, which is complex for some values of $\left.s\right)$, the closing of the gap means that the stationary distribution becomes degenerate for $|s|$ above the critical value in the limit $L \rightarrow \infty$. For $|s|$ below the critical value, the stationary probability $\mathbf{p}_{\text {stat }}^{s}=\mathbf{r}_{s, 0}^{D}$ is unique and one can check numerically that it has a reflection symmetry $y \rightarrow-y$ also possessed by the generator: $\mathcal{U} W_{\text {Doob }}^{s} \mathcal{U}^{T}=W_{\text {Doob }}^{s}, \mathcal{U} \mathbf{p}_{\text {stat }}^{s}=\mathbf{p}_{\text {stat }}^{s}$, where $\mathcal{U}$ is the permutation matrix that maps the lattice site at $(x, \pm y)$ into the one at $(x, \mp y)$. For the argument that follows, it is useful to decompose the stationary probability into its upper and lower halves, as $\mathbf{p}_{\text {stat }}^{s}=\left(\mathbf{p}_{\uparrow}^{s}+\mathbf{p}_{\downarrow}^{s}\right) / 2$, where $\mathbf{p}_{\uparrow}^{s}\left(\mathbf{p}_{\downarrow}^{s}\right)$ is zero for $y<0(y>0)$; both $\mathbf{p}_{\uparrow}^{s}$ and $\mathbf{p}_{\downarrow}^{s}$ are normalized, and $\mathcal{U} \mathbf{p}_{\uparrow}^{s}=\mathbf{p}_{\downarrow}^{s}\left(\mathcal{U} \mathbf{p}_{\downarrow}^{s}=\mathbf{p}_{\uparrow}^{s}\right)$. Beyond the critical point, the right eigenspace (corresponding to the space of stationary distributions) is spanned by $\mathbf{r}_{s, 0}^{D}=\left(\mathbf{p}_{\uparrow}^{s}+\mathbf{p}_{\downarrow}^{s}\right) / 2$ (which by continuity shares the symmetries of $\mathbf{p}_{\text {stat }}^{s}$ below the critical value), and an orthogonal stationary state numerically found to be $\mathbf{r}_{s, 1}^{D}=\left(\mathbf{p}_{\uparrow}^{s}-\mathbf{p}_{\downarrow}^{s}\right) / 2$, for which $\mathcal{U} \mathbf{r}_{s, 1}^{D}=-\mathbf{r}_{s, 1}^{D}$. More conveniently, one can use $\mathbf{p}_{\uparrow}^{s}$ and $\mathbf{p}_{\downarrow}^{s}$, which concentrate (more sharply as $|s|$ is increased) around the upper and lower edges of the slit, respectively, as a basis to form possible stationary distributions. A particle starts from an initial site, moves toward one of the slit edges, and remains there, as the probability of making the journey to the other edge is vanishingly small for $L \rightarrow \infty$. The $\mathbb{Z}_{2}$ reflection symmetry is spontaneously broken.

To conclude our exploration of the DPT, we use the inverse participation ratio (IPR) as an order parameter. This is defined as the sum of the squared probabilities of occupation in the stationary state over all sites, $\operatorname{IPR}(s)=\sum_{n}\left[p_{\text {stat }}^{s}(n)\right]^{2}$, and is widely employed in the characterization of localized states [56]. In idealized cases where the probability is evenly distributed over $\mathcal{N}$ sites and is zero outside, the IPR is $1 / \mathcal{N}$. For $s=0$ (flat solution), $\operatorname{IPR}(0)=1 / L^{2}$, as shown in Fig. 3(b). In the case of infinitely sharp bands, we would have IPR $=1 / h L^{2}$ for the inner band $\left[\mathrm{IPR}=1 /(1-h) L^{2}\right.$ for the outer band], and therefore band dynamics correspond to IPRs between $1 / L^{2}$ and $1 / h L^{2}\left[1 /(1-h) L^{2}\right]$, and larger values must arise from localized vortices. Accordingly, we set $\max \left\{1 / h L^{2}, 1 /(1-h) L^{2}\right\}$ as a threshold, then use $\mathbf{p}_{\text {stat }}^{s}$ to calculate the IPR for values that are smaller than such threshold, and otherwise base the calculation of the IPR on $\mathbf{p}_{\uparrow}^{s}=\mathbf{r}_{s, 0}^{D}+\mathbf{r}_{s, 1}^{D}$ (or $\mathbf{p}_{\downarrow}^{s}=\mathbf{r}_{s, 0}^{D}-\mathbf{r}_{s, 1}^{D}$, as they give equivalent results), as a particle is trapped in one slit edge. The argument works in the limit of large $L$, but we use our finite-size results as approximations to that limit. The IPR shown in the main panel of Fig. 3(b) reaches a small and stable value for $s$ below the critical point. As expected from the characteristics of band dynamics, multiplication of the IPR by $L^{2}$ in this range of $s$ leads to an excellent collapse; see the inset of Fig. 3(b). For large values of $|s|$, the IPR approaches a constant value of $1 / 4$ as $|s| \rightarrow \infty$. The latter $(\mathcal{N}=4)$ corresponds to the existence of a vortex around an edge slit, where the loop comprises four lattice sites. If instead of the stationary state $\mathbf{p}_{\uparrow}^{s}$ ( or $\mathbf{p}_{\downarrow}^{s}$ ) we naively take $\mathbf{r}_{s, 0}^{D}$, the IPR approaches a value of $1 / 8$ for large $|s|$, corresponding to the existence of two vortices at the edges $(\mathcal{N}=8)$, as in the right panels of Fig. 1(b), but sharper.

\section{CONCLUSIONS}

We have shown that the two-dimensional random walk conditioned on partial currents undergoes a DPT between delocalized bands and localized vortices, where particles condensate around the slit edges and a $\mathbb{Z}_{2}$ reflection symmetry is spontaneously broken. In principle, this DPT should be observable in experiments based on driven diffusive systems via an accurate characterization of the probability distribution of partial currents. While our analyses assume the presence of a sizable driving field, a change of field strength does not lead to any qualitatively different behavior. For zero field, however, we find that the band regime is absent from the dynamics. 
Intriguing localization phenomena have also been observed in other random walks, e.g., in the maximal entropy random walk on a lattice [57], which can also be understood as the result of conditioning on a particular observable [58], or in random walks on complex networks, where some DPTs have been also investigated [59]. Many interesting questions remain open, however, regarding, for example, the existence of similar localization effects when other processes (including exclusion effects or interactions) or number of spatial dimensions are considered. Whether such effects are relevant in dissipative quantum walks $[60,61]$ (where the spectral theory of Liouvillians can be brought to bear [55,62]) is also an interesting question, as an answer in the affirmative might stimulate a search for potential relationships to well-known localization effects in quantum mechanics $[63,64]$.

\section{ACKNOWLEDGMENTS}

We thank Rubén Hurtado-Gutiérrez and Pablo Hurtado for insightful discussions. The research leading to these results has received funding from the European Union's Horizon 2020 research and innovation programme under the Marie Sklodowska-Curie Cofund Programme Athenea3I Grant Agreement No. 754446, and from the Project of I+D+i Ref. PID2020-113681GB-I00, financed by MICIN/AEI/10.13039/501100011033 and FEDER "A way to make Europe". We are grateful for the the computing resources and related technical support provided by PROTEUS, the supercomputing center of Institute Carlos I in Granada, Spain, and by CRESCO/ENEAGRID High Performance Computing infrastructure and its staff [65], which is funded by ENEA, the Italian National Agency for New Technologies, Energy and Sustainable Economic Development and by Italian and European research programmes.

\section{APPENDIX A: MACROSCOPIC FLUCTUATION THEORY OF THE TWO-DIMENSIONAL RANDOM WALK}

The macroscopic fluctuation theory (MFT) is a powerful framework for the study of dynamical fluctuations of driven diffusive systems [15]. When such processes take place over a square region of linear size $L$, the probability of a trajectory of duration $T,\{\rho(\mathbf{r}, t), \mathbf{j}(\mathbf{r}, t)\}_{0}^{T}$, adopts a large deviation form

$$
P[\rho(\mathbf{r}, t), \mathbf{j}(\mathbf{r}, t)] \sim e^{-L^{2} I[\rho, \mathbf{j}]},
$$

where the so-called rate functional is as follows (the spatiotemporal dependence of $\rho$ and $\mathbf{j}$ is omitted for ease of notation)

$$
I[\rho, \mathbf{j}]=\int_{0}^{T} d t \int_{\Lambda} d \mathbf{r} \frac{[\mathbf{j}+D(\rho) \nabla \rho-\sigma(\rho) \mathbf{E}]^{2}}{2 \sigma(\rho)} .
$$

The spatial coordinates have been rescaled so that $\mathbf{r} \in \Lambda=$ $[-1 / 2,1 / 2] \times[-1 / 2,1 / 2]$ (i.e., a square region of unit area with the origin $(0,0)$ at its center). The probability is maximized around the macroscopic average current, which in the presence of a uniform driving field $\mathbf{E}$ is $\mathbf{j}=-D(\rho) \nabla \rho+$ $\sigma(\rho) \mathbf{E}$, where the diffusivity $D(\rho)$ and the mobility $\sigma(\rho)$ are in general functions of the density field.

For the particular case of a random walk, $D=1 / 4$ and $\sigma(\rho)=\rho / 2$, the functional can be expanded as

$$
\begin{aligned}
I[\rho, \mathbf{j}]= & \int_{0}^{T} d t \int_{\Lambda} d \mathbf{r} \frac{\left[\mathbf{j}+\frac{1}{4} \nabla \rho-\frac{1}{2} \rho \mathbf{E}\right]^{2}}{\rho}=\int_{0}^{T} d t \int_{\Lambda} d \mathbf{r} \frac{\mathbf{j}^{2}+\frac{1}{16}(\nabla \rho)^{2}}{\rho}+\frac{E^{2}}{4} \int_{0}^{T} d t \int_{\Lambda} d \mathbf{r} \rho \\
& +\frac{1}{2} \int_{0}^{T} d t \int_{\Lambda} d \mathbf{r} \frac{\mathbf{j} \cdot \nabla \rho}{\rho}-\int_{0}^{T} d t \mathbf{E} \cdot\left(\int_{\Lambda} d \mathbf{r} \mathbf{j}+\frac{1}{4} \int_{\Lambda} d \mathbf{r} \nabla \rho\right) .
\end{aligned}
$$

As we assume the system has periodic boundary conditions, the last term is $\int_{\Lambda} d \mathbf{r} \nabla \rho=0$, while the following integral can be simplified using integration by parts,

$$
\begin{aligned}
\int_{0}^{T} d t \int_{\Lambda} d \mathbf{r} \frac{\mathbf{j} \cdot \nabla \rho}{\rho} & =\int_{0}^{T} d t \int_{\Lambda} d \mathbf{r} \mathbf{j} \cdot \nabla \ln \rho=\int_{0}^{T} d t \int_{\Lambda} d \mathbf{r} \nabla \cdot(\mathbf{j} \ln \rho)-(\nabla \cdot \mathbf{j}) \ln \rho=\int_{0}^{T} d t \int_{\Lambda} d \mathbf{r}\left(\partial_{t} \rho\right) \ln \rho \\
& =A(T)-A(0),
\end{aligned}
$$

where we have taken into account that the density and current fields are coupled via the continuity equation $\partial_{t} \rho+\nabla \cdot \mathbf{j}=0$. The function $A(t)=\int_{\Lambda} d \mathbf{r}(\rho \ln \rho-\rho)$ is bounded in time, which distinguishes the corresponding term in $I[\rho, \mathbf{j}]$ from the other terms, as they are all time extensive. Considering that the system is closed with a fixed number of particles $N=\rho_{0} L^{2}$, by definition $\rho_{0}=\int_{\Lambda} d \mathbf{r} \rho$. Together with the definition of a global current $\mathbf{J}_{G}=\int_{\Lambda} d \mathbf{r} \mathbf{j}$, all of this leads to a simpler expression for the functional,

$$
\begin{aligned}
I[\rho, \mathbf{j}]= & \int_{0}^{T} d t \int_{\Lambda} d \mathbf{r} \frac{\mathbf{j}^{2}+\frac{1}{16}(\nabla \rho)^{2}}{\rho}+\frac{E^{2} T \rho_{0}}{4} \\
& +\frac{1}{2}(A(T)-A(0))-\mathbf{E} \cdot \int_{0}^{T} d t \mathbf{J}_{G}
\end{aligned}
$$

We next calculate by contraction from the MFT functional (A5) the probability of having a current $J$ through the slit, which reads

$$
P(J) \sim \exp \left\{-T L^{2} G(J)\right\}
$$

with $G(J)=\lim _{T \rightarrow \infty} \frac{1}{T} \min _{\{\rho, \mathbf{j}\}_{0}^{T}}^{*} I[\rho, \mathbf{j}]$. Here $*$ means that the minimization is subject to the constraints $J=T^{-1} \int_{0}^{T} d t \int_{-h / 2}^{h / 2} d y j_{x}(0, y ; t)$ and $\partial_{t} \rho=-\nabla \cdot \mathbf{j}$. This variational problem will be applied to the different dynamical regimes discussed in the main text, which are idealizations of the density and current fields observed in the stationary states of the Doob-transformed microscopic dynamics. Sketches of these idealized regimes are provided in Fig. 4. They all 
(a)

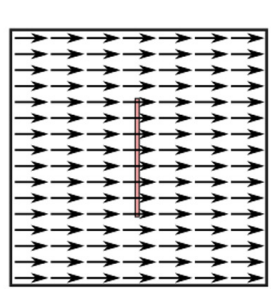

(b)

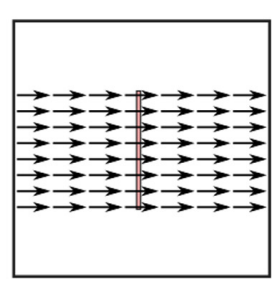

(c)

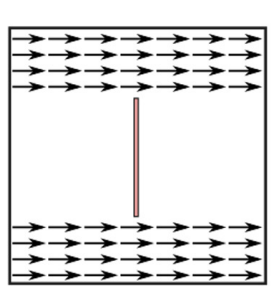

(d)

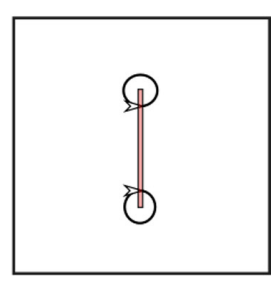

FIG. 4. Dynamical regimes for the two-dimensional random walk conditioned on different values of the partial current $J$ across a slit (vertical strip in the center). (a) Flat profile. (b) Inner band. (c) Outer band. (d) Vortices. These regimes are idealizations of those observed in the microscopic analysis based on the Doob transform.

correspond to time-independent density and current fields, for which the rescaled functional can be written as

$$
G(J)=\min _{\{\rho, \mathbf{j}\}}^{*}\left[\int_{\Lambda} d \mathbf{r} \frac{\mathbf{j}^{2}+\frac{1}{16}(\nabla \rho)^{2}}{\rho}+\frac{E^{2} \rho_{0}}{4}-\mathbf{E} \cdot \mathbf{J}_{G}\right] .
$$

The third term in (A5) has been neglected, since the discussion focuses on the long-time limit, and, as we pointed out before, $\lim _{T \rightarrow \infty}(A(T)-A(0)) / T=0$.

Different forms of the density and current fields conditioned on a given partial current $J$ will be evaluated in each particular regime, always focusing on situations where $\mathbf{E}$ is horizontal and points to the right, $\mathbf{E}=E \hat{\mathbf{x}}$. When a parameter characterizes an idealized regime, as embodied in a given ansatz for $\{\rho(\mathbf{r}), \mathbf{j}(\mathbf{r})\}$, the parameter choice that minimizes $G(J)$ for that value of $J$ is the one that is (overwhelmingly) more likely to be observed. When different ansätze, each one individually optimized by the appropriate parameter choice, are compared for a given $J$, the one that yields a minimum value of $G(J)$ is the one that would be observed in practice.

\section{APPENDIX B: FLAT SOLUTION}

For a given partial current $J$ across a slit of length $h$, a flat density profile that sustains a uniform current, see Fig. 4(a), takes the following values:

$$
\rho=\rho_{0}, \quad \mathbf{j}=\frac{J}{h} \hat{\mathbf{x}}
$$

The time-rescaled MFT functional (A7) takes a simple quadratic form:

$$
G_{\text {flat }}(J)=\frac{J^{2}}{h^{2} \rho_{0}}+\frac{E^{2} \rho_{0}}{4}-\frac{E J}{h}=\rho_{0}\left(\frac{J}{h \rho_{0}}-\frac{E}{2}\right)^{2} .
$$

The fluctuations around the mean value $\langle J\rangle=\frac{E h \rho_{0}}{2}$ are thus Gaussian, with a variance that decreases as $1 / T$,

$$
P_{\text {flat }}(J) \sim \exp \left[-(J-\langle J\rangle)^{2} /\left(\frac{h^{2} \rho_{0}}{L^{2} T}\right)\right] .
$$

\section{APPENDIX C: INNER BAND SOLUTION}

We next analyze the case when the density profile forms a horizontal band that decays for $|y|>h / 2$, and the current is proportional to the density. For simplicity, we assume an exponential decay:

$$
\rho(x, y)= \begin{cases}C e^{-(y-h / 2) / \ell} & y>h / 2 \\ C & |y| \leqslant h / 2, \quad \mathbf{j}(x, y)=j_{0} \rho(x, y) \hat{\mathbf{x}} . \\ C e^{(y+h / 2) / \ell} & y<-h / 2\end{cases}
$$

In this case, there is one parameter in the solution, which is the characteristic length of the exponential decay $\ell$. In Fig. 4(b), this regime is illustrated for $\ell \ll h$; there the density profile as a function of $y$ approaches a step function. Both density and current are invariant under translations along the horizontal axis.

In order to determine $C$, we perform the integral of the density over the square region $\Lambda$ and equate it to $\rho_{0}$ :

$$
\begin{aligned}
\rho_{0} & =\int_{-1 / 2}^{1 / 2} d y \rho(x, y)=C\left(2 \ell\left(1-e^{-(1-h) / 2 \ell}\right)+h\right) \\
& \Rightarrow C=\frac{\rho_{0}}{2 \ell\left(1-e^{-(1-h) / 2 \ell}\right)+h} .
\end{aligned}
$$

In the following, it will be useful to divide the integrated density into two contributions, namely, that corresponding to particles passing through the slit $\rho_{\text {in }}(\ell)$, which is decreasing in $\ell$, and that corresponding to particles moving across the region where $|y|>h / 2, \rho_{\text {out }}(\ell)$, which increases with $\ell$,

$$
\begin{gathered}
\rho_{\text {in }}(\ell)=C h=\frac{\rho_{0} h}{2 \ell\left(1-e^{-(1-h) / 2 \ell}\right)+h}, \\
\rho_{\text {out }}(\ell)=\rho_{0}-\rho_{\text {in }}(\ell)=\frac{\rho_{0} 2 \ell\left(1-e^{-(1-h) / 2 \ell}\right)}{2 \ell\left(1-e^{-(1-h) / 2 \ell}\right)+h} .
\end{gathered}
$$

As for the proportionality constant $j_{0}$, it can be obtained from the fact that the current integrated over the slit is by definition $J$ :

$$
J=\int_{-h / 2}^{h / 2} d y j(x, y)=j_{0} \rho_{\text {in }}(\ell), \quad j_{0}=\frac{J}{\rho_{\text {in }}(\ell)} .
$$

The rate functional (A7) thus takes the form

$$
\begin{aligned}
G_{\text {i.b. }}(J, \ell) & =\int_{\Lambda} d \mathbf{r} \frac{j_{0}^{2} \rho^{2}+\frac{1}{16}\left(\partial_{y} \rho\right)^{2}}{\rho}+\frac{E^{2} \rho_{0}}{4}-E j_{0} \rho_{0} \\
& =\rho_{0}\left(\frac{J}{\rho_{\text {in }}(\ell)}-\frac{E}{2}\right)^{2}+\frac{\rho_{\text {out }}(\ell)}{16 \ell^{2}} .
\end{aligned}
$$

The first term quantifies the cost of having a density $\rho_{\text {in }}(\ell)$ different from $2 J / E$, and the second that of forming a nonuniform density field. The flat solution previously discussed corresponds to $\rho_{\text {in }}(\ell)=\rho_{0} h$, and hence the first term is minimized for $J=E h \rho_{0} / 2=\langle J\rangle$ in that case. As the density is 
(a)

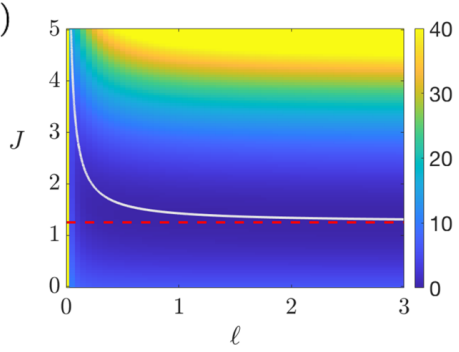

(b)

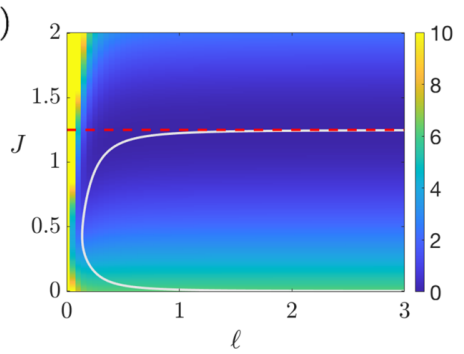

(c)

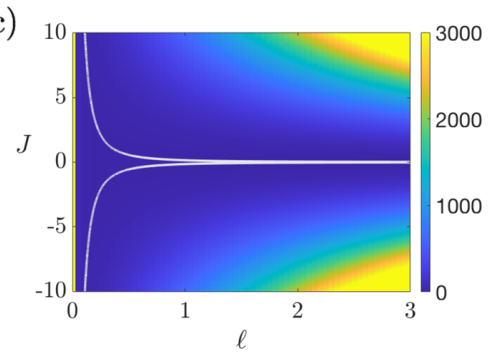

FIG. 5. Macroscopic functionals for the inner band, the outer band, and the vortex solution as functions of the characteristic length $\ell$ and the partial current $J$. (a) Inner-band functional $G_{\text {i.b. }}(J, \ell)$. (b) Outer-band functional $G_{\text {o.b. }}(J, \ell)$. (a) Vortex functional $G_{\text {vort. }}(J, \ell)$. In each case, the location of the minimum value of the functional for each $J$ is shown as a gray continuous line. In panels (a) and (b), the red discontinuous line corresponds to the average partial current $\langle J\rangle$.

then uniform, $\ell \rightarrow \infty$, the second term proportional to $\rho_{\text {out }}(\ell)$ also vanishes.

Of all possible band profiles, which are characterized by different values of the characteristic length $\ell$, the one that is most likely to be observed for a given $J$ is the one that minimizes the functional. For fluctuations around the mean value, we have two possibilities:

(1) If $J>\langle J\rangle$, a band is formed whose vertical profile is steeper as $J$ increases, as, in order to minimize the first term, we need some $\rho_{\text {in }}(\ell)>\rho_{0} h$, which requires having a finite value of $\ell$ (nonflat solution), so $\rho_{\text {out }}(\ell)<\rho_{0}(1-h)$.

(2) If $0<J \leqslant\langle J\rangle$, we still have a flat solution, as $\rho_{0} h$ is the lowest value that $\rho_{\text {in }}(\ell)$ can achieve, and moreover the second term vanishes for such flat solution, $\ell \rightarrow \infty$.

For $J>\langle J\rangle$, there is an effective competition between the first and the second terms in Eq. (C5), as the second, $\rho_{\text {out }}(\ell) / 16 \ell^{2}$, includes the cost of creating a nonuniform density profile, and is a decreasing function of $\ell$. In the limit of very large $J$, the first term clearly dominates and the system adopts an abrupt vertical profile, $\ell \approx 0$, but intermediate cases between $\langle J\rangle$ and such large values of $J$ must be determined from a minimization of $G_{\text {i.b. }}(J, \ell)$.

Given in terms of $\ell$, Eq. (C5) is too cumbersome for a convenient analytical minimization. Instead, the values of $\ell$ that minimize this function have been found numerically. In Fig. 5(a), we provide a surface plot showing $G_{\text {i.b. }}(J, \ell)$ as a function of $J$ and $\ell$ for $E=5$ and $h=1 / 2$. The values of $\ell$ where the minimum is achieved for each $J$ are displayed as a gray continuous line. As expected, it turns out that for $0<J \leqslant\langle J\rangle$ the minimum is achieved for $\ell \rightarrow \infty$, and for $J>\langle J\rangle$ the position of the minimum gets closer and closer to zero as $J$ increases. $\langle J\rangle$ is highlighted by a red discontinuous line.

\section{APPENDIX D: OUTER BAND SOLUTION}

We next consider the case when the density profile forms a horizontal band that avoids passing through the slit, which is constant for $|y|>h / 2$ and decays for $|y| \leqslant h / 2$, see Fig. 4(c), where this regime is illustrated for $\ell \ll h$. As in the previous case, the current is taken to be proportional to the density. For simplicity, we again assume an exponential decay:

$$
\begin{aligned}
\rho(x, y) & = \begin{cases}C & y>h / 2 \\
\frac{C}{1+e^{-h / \ell}}\left(e^{(y-h / 2) / \ell}+e^{-(y+h / 2) / \ell}\right) & |y| \leqslant h / 2, \\
C & y<-h / 2\end{cases} \\
\mathbf{j}(x, y) & =j_{0} \rho(x, y) \hat{\mathbf{x}},
\end{aligned}
$$

where the prefactor of the middle term has been chosen so as to ensure continuity. In order to determine $C$, we need to normalize the integral of the density over the square region $\Lambda$ and equate it to $\rho_{0}$ :

$$
\begin{aligned}
\rho_{0} & =\int_{-1 / 2}^{1 / 2} d y \rho(x, y)=C\left(1-h+2 \ell \tanh \left(\frac{h}{2 \ell}\right)\right) \\
& \Rightarrow C=\frac{\rho_{0}}{1-h+2 \ell \tanh \left(\frac{h}{2 \ell}\right)} .
\end{aligned}
$$

As in the previous case, it will be useful to divide the integrated density into two contributions that corresponding to particles passing through the slit $\rho_{\text {in }}(\ell)$, which is now increasing in $\ell$, and that corresponding to particles moving across the region where $|y|>h / 2, \rho_{\text {out }}(\ell)$, which decreases with $\ell$,

$$
\begin{aligned}
& \rho_{\text {out }}(\ell)=C(1-h)=\frac{\rho_{0}(1-h)}{1-h+2 \ell \tanh \left(\frac{h}{2 \ell}\right)}, \\
& \rho_{\text {in }}(\ell)=\rho_{0}-\rho_{\text {out }}(\ell)=\frac{\rho_{0} 2 \ell \tanh \left(\frac{h}{2 \ell}\right)}{1-h+2 \ell \tanh \left(\frac{h}{2 \ell}\right)} .
\end{aligned}
$$

As for the proportionality constant $j_{0}$, it can be determined from the fact that the current integrated over the slit is by definition $J$ :

$$
J=\int_{-h / 2}^{h / 2} d y j(x, y)=j_{0} \rho_{\text {in }}(\ell), \quad j_{0}=\frac{J}{\rho_{\text {in }}(\ell)} .
$$

The rate functional is in this case

$$
G_{\text {o.b. }}(J, \ell)=\int_{\Lambda} d \mathbf{r} \frac{j_{0}^{2} \rho^{2}+\frac{1}{16}\left(\partial_{y} \rho\right)^{2}}{\rho}+\frac{E^{2} \rho_{0}}{4}-E j_{0} \rho_{0}=\rho_{0}\left(\frac{J}{\rho_{\text {in }}(\ell)}-\frac{E}{2}\right)^{2}+\frac{\rho_{0}\left(\sinh \left(\frac{h}{2 \ell}\right)+\arctan e^{-h / 2 \ell}-\arctan e^{h / 2 \ell}\right)}{4 \ell e^{h / 2 \ell}\left(1+e^{-h / \ell}\right)\left(1-h+2 \ell \tanh \left(\frac{h}{2 \ell}\right)\right)} .
$$


Again, the first term quantifies the cost of having a density $\rho_{\text {in }}(\ell)$ different from $2 J / E$, and the second that of forming a nonuniform density field. The flat solution is given by $\rho_{\text {in }}(\ell)=\rho_{0} h$, and hence the first term is minimized for $J=\langle J\rangle$ in that case. This corresponds to a uniform density, $\ell \rightarrow \infty$, for which the second term also vanishes.

Of all possible band profiles, which are characterized by different values of the characteristic length $\ell$, the one that is most likely to be observed for a given $J$, is the one that minimizes the functional. For fluctuations around the mean value, we have two possibilities:

(1) If $0<J<\langle J\rangle$, a band is formed whose vertical profile is steeper as $J$ decreases from $\langle J\rangle$, at least up to certain value of $J$ (more about this later), as, in order to minimize the first term, we need some $\rho_{\text {in }}(\ell)<\rho_{0} h$, which requires having a finite value of $\ell$ (nonflat solution), so $\rho_{\text {out }}(\ell)>\rho_{0}(1-h)$.

(2) If $J \geqslant\langle J\rangle$, we have a flat solution, as $\rho_{0} h$ is the highest value that $\rho_{\text {in }}(\ell)$ can achieve, and moreover the second term vanishes for such flat solution, $\ell \rightarrow \infty$.

For $0<J<\langle J\rangle$ there is an effective competition between the first and the second terms in Eq. (D5), as the second includes the cost creating a nonuniform density profile, and is a decreasing function of $\ell$. As $J$ decreases from the average value $\langle J\rangle$, the first term tries to adapt to a smaller $\rho_{\text {in }}(\ell)$, so $\ell$ becomes smaller, at the cost of creating a nonuniform profile, which contributes to the second term of $G_{\text {o.b. }}(J, \ell)$. However, when $J$ gets very small, deviations around the average in the first term become less important than the second term, and therefore we again obtain a flat solution, $\ell \rightarrow \infty$.
The values of $\ell$ that minimize $G_{\text {o.b. }}(J, \ell)$ have been found numerically. In Fig. 5(b), we provide a surface plot showing $G_{\text {o.b. }}(J, \ell)$ as a function of $J$ and $\ell$ for $E=5$ and $h=1 / 2$. The values of $\ell$ where the minimum value is achieved for each $J$ are displayed as a gray continuous line. As expected, it turns out that for $J>\langle J\rangle$ the minimum is always achieved for $\ell \rightarrow$ $\infty$, and for $0<J \leqslant\langle J\rangle$ the value of $\ell$ where the minimum is achieved has the nonmonotonic behavior discussed in the previous paragraph. $\langle J\rangle$ is highlighted by a red discontinuous line.

\section{APPENDIX E: VORTICES}

Finally, we consider a solution where vortices form around the edges of the slit, see Fig. 4(d), which as mentioned in the main text resembles a configuration previously described in the two-dimensional simple exclusion process conditioned on partial currents [45]. Due to the rotational symmetry of the solution, the density is best represented in polar coordinates, by some decreasing function of the radial distance $r$ from the center of the vortex. We again assume the density profile to be of exponential form due to its simplicity:

$$
\rho(r, \varphi)= \begin{cases}0 & r<\tilde{r}, \\ C e^{-(r-\tilde{r}) / \ell} & r \geqslant \tilde{r} .\end{cases}
$$

Again the steepness of the profile is given by the characteristic length $\ell$. The role played by the cutoff radius $\tilde{r}$ will be discussed later. The normalization in this case is given by

$$
\rho_{0}=2 \int_{0}^{2 \pi} d \varphi \int_{\tilde{r}}^{\infty} d r r C e^{-(r-\tilde{r}) / \ell}=4 \pi C \ell(\tilde{r}+\ell) \Rightarrow C=\frac{\rho_{0}}{4 \pi \ell(\tilde{r}+\ell)} .
$$

The factor of 2 multiplying the integral of the density arises from the existence of two vortices, each one located in the vicinity of each of the two edges of the slit. The upper limit of integration in the integral over $r$ should not exceed $h / 2$, but at least for $\ell \ll h$ the simplification arising from taking it to infinity is expected to compensate for any slight loss of accuracy, as we will explain below.

The current at the vortices in the microscopic results was numerically found to be roughly proportional to the density divided by $r$. For simplicity, we assume a strict proportionality $j(r) \propto \rho(r) / r$ in our ansatz:

$$
\mathbf{j}(r)=j_{0} \frac{\rho(r)}{r} \hat{\varphi}= \begin{cases}\mathbf{0} & r<\tilde{r}, \\ \frac{j_{0} C e^{-(r-\tilde{r}) / \ell}}{r} \hat{\varphi} & r \geqslant \tilde{r} .\end{cases}
$$

The proportionality factor $j_{0}$ is as usual determined from a calculation of the partial current through the slit

$$
\begin{aligned}
J & =2 \int_{\tilde{r}}^{\infty} d r j(r)=2 j_{0} C e^{\tilde{r} / \ell} \Gamma(0, \tilde{r} / \ell) \\
& \Rightarrow j_{0}=\frac{J 2 \pi \ell(\tilde{r}+\ell) e^{-\tilde{r} / \ell}}{\rho_{0} \Gamma(0, \tilde{r} / \ell)},
\end{aligned}
$$

where the final expression contains an incomplete gamma function evaluation.
The rate functional is in this case

$$
\begin{aligned}
G_{\text {vort. }}(J, \ell) & =\int_{\Lambda} d \mathbf{r} \frac{\mathbf{j}^{2}+\frac{1}{16}\left(\partial_{r} \rho\right)^{2}}{\rho}+\frac{E^{2} \rho_{0}}{4}-\mathbf{E} \cdot \mathbf{J}_{G} \\
& =\frac{J^{2} 4 \pi^{2} \ell(\tilde{r}+\ell) e^{-\tilde{r} / \ell}}{\rho_{0} \Gamma(0, \tilde{r} / \ell)}+\frac{\rho_{0}}{8 \ell^{2}}+\frac{E^{2} \rho_{0}}{4},
\end{aligned}
$$

where we have taken into consideration that, due to the vortex geometry, the total current vanishes $\mathbf{J}_{G}=\mathbf{0}$.

The values of $\ell$ that minimize $G_{\text {vort. }}(J, \ell)$ have been found numerically. In Fig. 5(c), we provide a surface plot showing $G_{\text {vort. }}(J, \ell)$ as a function of $J$ and $\ell$ for $E=5$ and $h=1 / 2$. The values of $\ell$ where the minimum value is achieved for each $J$ are displayed as a gray continuous line. We see that the $\ell$ that minimizes $G_{\text {vort. }}(J, \ell)$ decreases as $|J|$ increases. For $|J| \gtrsim 2$, the condition $\ell \ll h$ that was assumed before in order to simplify certain integrals is indeed satisfied. In the figure, we have set the cutoff $\tilde{r}=0.001$, as the qualitative dependence on $\ell$ is independent of this choice. As for the quantitative influence of the cutoff $\tilde{r}$ on the minimum value that is achieved by $G_{\text {vort. }}(J, \ell)$ for a given $J$, it will be discussed next when the MFT functionals of the four dynamical regimes that have been considered (that is, flat solution, inner band, outer band, and vortices) are compared over a range of fluctuations of the partial current $J$. 

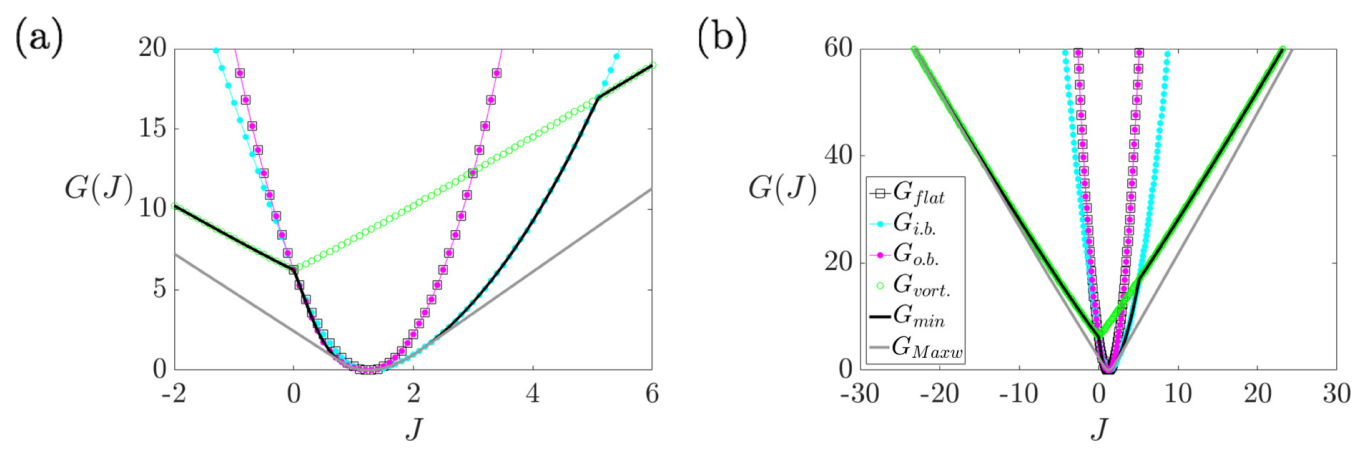

FIG. 6. Comparison of the MFT functional for the different dynamical regimes under consideration. The MFT functional of the flat solution $G_{\text {flat }}(J)$, the inner-band solution $G_{\text {i.b. }}\left(J, \ell^{*}(J)\right)$, the outer-band solution $G_{\text {o.b. }}\left(J, \ell^{*}(J)\right)$, and the vortex solution $G_{\text {vort. }}\left(J, \ell^{*}(J)\right)$ are displayed. The minimum value among them corresponding to each $J$, which is denoted $G_{\min .}(J)$, is also shown, as well as its convex envelope $G_{\mathrm{Maxw}}(J)$. Panels (a) and (b) contain the same results with significantly different axis ranges, thus making it possible to inspect different aspects of them (see text for an explanation). Figure 1(c) displays the same results with yet another choice of axis ranges.

\section{APPENDIX F: COMPARISON OF DIFFERENT DYNAMICAL REGIMES}

In order to determine the most likely form of a fluctuation away from the mean, $J \neq\langle J\rangle$, the functionals corresponding to the different dynamical regimes must be compared. This includes the flat solution $G_{\text {flat }}(J)$, the inner-band solution $G_{\text {i.b. }}\left(J, \ell^{*}(J)\right)$, the outer-band solution $G_{\text {o.b. }}\left(J, \ell^{*}(J)\right)$, and the vortex solution $G_{\text {vort. }}\left(J, \ell^{*}(J)\right)$, where $\ell^{*}(J)$ denotes the value of $\ell$ for which each of those functionals are minimized for a given $J$, which was highlighted by gray continuous lines in the different panels of Fig. 5. The dependence on $\ell^{*}(J)$ is omitted in the main text, as the role played by the characteristic length $\ell$ is not discussed there.

A comparison of the different dynamical regimes is provided in Fig. 6. Aside from the functionals corresponding to the different ansätze, we include the functional that takes the minimum value at each $J, G_{\min }(J)=$ $\min \left\{G_{\text {flat }}(J), G_{\text {i.b. }}\left(J, \ell^{*}(J)\right), G_{\text {o.b. }}\left(J, \ell^{*}(J)\right), G_{\text {vort. }}\left(J, \ell^{*}(J)\right)\right\}$, and also its convex envelope $G_{\text {Maxw. }}(J)$. While $G_{\text {min. }}(J)$, from which the macroscopic SCGF of Fig. 2(b) has been obtained via a Legendre-Fenchel transform, maximizes the probability for each value of $J$, it is not convex. If the inverse LegendreFenchel transform is performed on the SCGF, we then obtain $G_{\text {Maxw. }}(J)$, which is convex. This Maxwell construction highlights a coexistence between the different dynamical phases [16].

The two panels of Fig. 6 show essentially the same results, which were moreover displayed in Fig. 1(c), but the axis ranges are very different and thus focus on different aspects. In Fig. 6(a), which is meant to facilitate the comparison between the different dynamical regimes, we can clearly see that $G_{\min .}(J)$ takes values across the range of $J$ as follows:

$$
G_{\text {min. }}(J)= \begin{cases}G_{\text {vort. }}\left(J, \ell^{*}(J)\right) & J<0, \\ G_{\text {o.b. }}\left(J, \ell^{*}(J)\right) & 0 \leqslant J<\langle J\rangle, \\ G_{\text {flat }}(J) & J=\langle J\rangle, \\ G_{\text {i.b. }}\left(J, \ell^{*}(J)\right) & \langle J\rangle<J<\tilde{J}, \\ G_{\text {vort. }}\left(J, \ell^{*}(J)\right) & \tilde{J} \leqslant J .\end{cases}
$$

The average $\langle J\rangle$ corresponds to the absolute minimum of $G_{\min .}(J)$ where the flat solution and the band solutions are equal, $G_{\min .}(\langle J\rangle)=G_{\text {flat }}(\langle J\rangle)=G_{\text {i.b. }}\left(\langle J\rangle, \ell^{*}(\langle J\rangle)\right)=$ $G_{\text {o.b. }}\left(\langle J\rangle, \ell^{*}(\langle J\rangle)\right)$, as expected from the discussions above. All solutions take the value $G_{\text {flat }}(0)=\rho_{0} E^{2} / 4$ for $J=0$, including $G_{\text {vort. }}\left(J, \ell^{*}(J)\right)$, which dominates immediately to the left, i.e., for $J<0$-corresponding to clockwise rotation in the upper edge and counterclockwise rotation in the lower edge of the slit-and also for $J \geqslant \tilde{J}$-corresponding to counterclockwise rotation in the upper edge and clockwise rotation in the lower edge of the slit-where $\tilde{J}$ is to some extent dependent on the choice of the cutoff $\tilde{r}$. Indeed, $G_{\text {vort. }}\left(J, \ell^{*}(J)\right)$ for different values of $\tilde{r}$ is qualitatively similar, starting from the same value at $J=0$, and growing symmetrically to right and left. However, the steepness of the increase as $|J|$ grows decreases as $\tilde{r}$ is made smaller, which in $G_{\text {min. }}(J)$ is only reflected in a slower growth for $J<0$ or $J>\tilde{J}$, and in a leftwards shift of $\tilde{J}$.

In Fig. 6(b), $G_{\text {Maxw. }}(J)$ is shown to arise from a coexistence of the outer-band solution and the vortex solution for $J<\langle J\rangle$, and from a coexistence of the inner-band solution and the vortex solution for $J>\langle J\rangle$. Such coexistences, which seem to be consistent with the superpositions of bands and vortices observed in the Doob-transformed microscopic dynamics of Fig. 1(b), should not be interpreted literally as signaling the existence of a first-order DPT in the random walks. We are considering a limited subset of idealized dynamical behaviors, and what is here described by a coexistence of them may in fact correspond to dynamical behaviors that are not fully describable in terms of the ansätze under consideration. In fact, our $G_{\min }(J)$ is an upper bound of the actual macroscopic functional restricted to a quite limited subset of density and current fields $\{\rho(\mathbf{r}), \mathbf{j}(\mathbf{r})\}$ (though qualitatively representative of some of the main features that are observed in the microscopic analysis), as is frequently the case is the analysis of mean-field solutions of dynamical large deviations of manybody systems [35]. Yet, despite its simplicity, our study does provide a sound qualitative understanding of the dynamical regimes under consideration - if not of the nature of the phase transition, which is actually continuous, as explained in the main text. 
[1] T. Bodineau and B. Derrida, Current Fluctuations in Nonequilibrium Diffusive Systems: An Additivity Principle, Phys. Rev. Lett. 92, 180601 (2004).

[2] T. Bodineau and B. Derrida, Current large deviations for asymmetric exclusion processes with open boundaries, J. Stat. Phys. 123, 277 (2006).

[3] B. Derrida, Non-equilibrium steady states: Fluctuations and large deviations of the density and of the current, J. Stat. Mech. (2007) P07023.

[4] D. J. Evans, E. G. D. Cohen, and G. P. Morriss, Probability of Second Law Violations in Shearing Steady States, Phys. Rev. Lett. 71, 2401 (1993).

[5] G. Gallavotti and E. G. D. Cohen, Dynamical Ensembles in Nonequilibrium Statistical Mechanics, Phys. Rev. Lett. 74, 2694 (1995).

[6] J. Kurchan, Fluctuation theorem for stochastic dynamics, J. Phys. A 31, 3719 (1998).

[7] J. L. Lebowitz and H. Spohn, A Gallavotti-Cohen-type symmetry in the large deviation functional for stochastic dynamics, J. Stat. Phys. 95, 333 (1999).

[8] C. Maes, The fluctuation theorem as a Gibbs property, J. Stat. Phys. 95, 367 (1999).

[9] R. J. Harris and G. M. Schuetz, Fluctuation theorems for stochastic dynamics, J. Stat. Mech. (2007) P07020.

[10] J. P. Garrahan, R. L. Jack, V. Lecomte, E. Pitard, K. van Duijvendijk, and F. van Wijland, Dynamical First-Order Phase Transition in Kinetically Constrained Models of Glasses, Phys. Rev. Lett. 98, 195702 (2007).

[11] J. P. Garrahan, R. L. Jack, V. Lecomte, E. Pitard, K. van Duijvendijk, and F. van Wijland, First-order dynamical phase transition in models of glasses: An approach based on ensembles of histories, J. Phys. A 42, 075007 (2009).

[12] J. P. Garrahan and I. Lesanovsky, Thermodynamics of Quantum Jump Trajectories, Phys. Rev. Lett. 104, 160601 (2010).

[13] F. Carollo, J. P. Garrahan, I. Lesanovsky, and C. PérezEspigares, Fluctuating hydrodynamics, current fluctuations, and hyperuniformity in boundary-driven open quantum chains, Phys. Rev. E 96, 052118 (2017).

[14] F. Carollo, J. P. Garrahan, I. Lesanovsky, and C. PérezEspigares, Making rare events typical in Markovian open quantum systems, Phys. Rev. A 98, 010103(R) (2018).

[15] L. Bertini, A. De Sole, D. Gabrielli, G. Jona-Lasinio, and C. Landim, Macroscopic fluctuation theory, Rev. Mod. Phys. 87, 593 (2015).

[16] H. Touchette, The large deviation approach to statistical mechanics, Phys. Rep. 478, 1 (2009).

[17] V. Lecomte, C. Appert-Rolland, and F. van Wijland, Thermodynamic formalism for systems with Markov dynamics, J. Stat. Phys. 127, 51 (2007).

[18] H. B. Callen, Thermodynamics and an Introduction to Thermostatistics, 2nd ed. (John Wiley \& Sons, New York, 1985).

[19] D. Simon, Construction of a coordinate Bethe Ansatz for the asymmetric simple exclusion process with open boundaries, J. Stat. Mech. (2009) P07017.

[20] V. Popkov, G. M. Schütz, and D. Simon, ASEP on a ring conditioned on enhanced flux, J. Stat. Mech. (2010) P10007.

[21] R. L. Jack and P. Sollich, Large deviations and ensembles of trajectories in stochastic models, Prog. Theor. Phys. Supp. 184, 304 (2010).
[22] R. Chetrite and H. Touchette, Nonequilibrium Microcanonical and Canonical Ensembles and Their Equivalence, Phys. Rev. Lett. 111, 120601 (2013).

[23] R. Chetrite and H. Touchette, Nonequilibrium Markov processes conditioned on large deviations, Ann. Henri Poincare 16, 2005 (2015).

[24] L. Bertini, A. De Sole, D. Gabrielli, G. Jona-Lasinio, and C. Landim, Current Fluctuations in Stochastic Lattice Gases, Phys. Rev. Lett. 94, 030601 (2005).

[25] T. Bodineau and B. Derrida, Distribution of current in nonequilibrium diffusive systems and phase transitions, Phys. Rev. E 72, 066110 (2005).

[26] L. Bertini, A. De Sole, D. Gabrielli, G. Jona-Lasinio, and C. Landim, Nonequilibrium current fluctuations in stochastic lattice gases, J. Stat. Phys. 123, 237 (2006).

[27] P. I. Hurtado and P. L. Garrido, Spontaneous Symmetry Breaking at the Fluctuating Level, Phys. Rev. Lett. 107, 180601 (2011).

[28] C. P. Espigares, P. L. Garrido, and P. I. Hurtado, Dynamical phase transition for current statistics in a simple driven diffusive system, Phys. Rev. E 87, 032115 (2013).

[29] R. L. Jack, I. R. Thompson, and P. Sollich, Hyperuniformity and Phase Separation in Biased Ensembles of Trajectories for Diffusive Systems, Phys. Rev. Lett. 114, 060601 (2015).

[30] O. Tsobgni Nyawo and H. Touchette, A minimal model of dynamical phase transition, EPL 116, 50009 (2016).

[31] Y. Baek, Y. Kafri, and V. Lecomte, Dynamical Symmetry Breaking and Phase Transitions in Driven Diffusive Systems, Phys. Rev. Lett. 118, 030604 (2017).

[32] R. J. Harris and H. Touchette, Phase transitions in large deviations of reset processes, J. Phys. A 50, 10LT01 (2017).

[33] A. Lazarescu, Generic dynamical phase transition in onedimensional bulk-driven lattice gases with exclusion, J. Phys. A 50, 254004 (2017).

[34] C. Pérez-Espigares, F. Carollo, J. P. Garrahan, and P. I. Hurtado, Dynamical criticality in open systems: Nonperturbative physics, microscopic origin, and direct observation, Phys. Rev. E 98, 060102(R) (2018).

[35] C. Pérez-Espigares, I. Lesanovsky, J. P. Garrahan, and R. Gutiérrez, Glassy dynamics due to a trajectory phase transition in dissipative Rydberg gases, Phys. Rev. A 98, 021804(R) (2018).

[36] C. Pérez-Espigares and P. I. Hurtado, Sampling rare events across dynamical phase transitions, Chaos 29, 083106 (2019).

[37] M. C. Bañuls and J. P. Garrahan, Using Matrix Product States to Study the Dynamical Large Deviations of Kinetically Constrained Models, Phys. Rev. Lett. 123, 200601 (2019).

[38] R. Hurtado-Gutiérrez, F. Carollo, C. Pérez-Espigares, and P. I. Hurtado, Building Continuous Time Crystals from Rare Events, Phys. Rev. Lett. 125, 160601 (2020).

[39] P. I. Hurtado, C. Pérez-Espigares, J. J. del Pozo, and P. L. Garrido, Symmetries in fluctuations far from equilibrium, Proc. Natl. Acad. Sci. U.S.A. 108, 7704 (2011).

[40] R. Villavicencio-Sanchez, R. J. Harris, and H. Touchette, Fluctuation relations for anisotropic systems, EPL 105, 30009 (2014).

[41] C. Pérez-Espigares, P. L. Garrido, and P. I. Hurtado, Weak additivity principle for current statistics in $d$ dimensions, Phys. Rev. E 93, 040103(R) (2016). 
[42] N. Tizón-Escamilla, C. Pérez-Espigares, P. L. Garrido, and P. I. Hurtado, Order and Symmetry Breaking in the Fluctuations of Driven Systems, Phys. Rev. Lett. 119, 090602 (2017).

[43] R. L. Jack, T. Nemoto, and V. Lecomte, Dynamical phase coexistence in the Fredrickson-Andersen model, J. Stat. Mech. (2020) 053204.

[44] C. Casert, T. Vieijra, S. Whitelam, and I. Tamblyn, Dynamical Large Deviations of Two-Dimensional Kinetically Constrained Models Using a Neural-Network State Ansatz, Phys. Rev. Lett. 127, 120602 (2021).

[45] T. Bodineau, B. Derrida, and J. L. Lebowitz, Vortices in the two-dimensional simple exclusion process, J. Stat. Phys. 131, 821 (2008).

[46] N. Kumar, H. Soni, S. Ramaswamy, and A. K. Sood, Anisotropic isometric fluctuation relations in experiment and theory on a self-propelled rod, Phys. Rev. E 91, 030102(R) (2015).

[47] G. Falasco, R. Pfaller, A. P. Bregulla, F. Cichos, and K. Kroy, Exact symmetries in the velocity fluctuations of a hot Brownian swimmer, Phys. Rev. E 94, 030602(R) (2016).

[48] S. Ciliberto and C. Laroche, An experimental test of the Gallavotti-Cohen fluctuation theorem, J. Phys. IV France 8, 215 (1998).

[49] K. Feitosa and N. Menon, Fluidized Granular Medium as an Instance of the Fluctuation Theorem, Phys. Rev. Lett. 92, 164301 (2004).

[50] The normalization of the Doob-generator eigenvectors is such that the largest absolute value of the components of $\mathbf{l}_{s, i}^{D}$ is one, and $\left(\mathbf{l}_{s, i}^{D}\right)^{T} \mathbf{r}_{s, i}^{D}=1$. Therefore, $\mathbf{l}_{s, 0}^{D}=(1,1, \ldots, 1)^{T}$ and the sum of all the components of $\mathbf{r}_{s, 0}^{D}$ is one ( $\mathbf{p}_{\text {stat }}^{s}=\mathbf{r}_{s, 0}^{D}$ is a probability vector).

[51] R. Gutiérrez and C. Pérez-Espigares, Generalized optimal paths and weight distributions revealed through the large deviations of random walks on networks, Phys. Rev. E 103, 022319 (2021).

[52] R. Villavicencio-Sanchez, R. J. Harris, and H. Touchette, Current loops and fluctuations in the zero-range process on a diamond lattice, J. Stat. Mech. (2012) P07007.
[53] H. Spohn, Large Scale Dynamics of Interacting Particles, Theoretical and Mathematical Physics (Springer, Berlin, 2012).

[54] B. Gaveau and L. S. Schulman, Multiple phases in stochastic dynamics: Geometry and probabilities, Phys. Rev. E 73, 036124 (2006).

[55] F. Minganti, A. Biella, N. Bartolo, and C. Ciuti, Spectral theory of Liouvillians for dissipative phase transitions, Phys. Rev. A 98, 042118 (2018).

[56] D. J. Thouless, Electrons in disordered systems and the theory of localization, Phys. Rep. 13, 93 (1974).

[57] Z. Burda, J. Duda, J. M. Luck, and B. Waclaw, Localization of the Maximal Entropy Random Walk, Phys. Rev. Lett. 102, 160602 (2009).

[58] F. Coghi, J. Morand, and H. Touchette, Large deviations of random walks on random graphs, Phys. Rev. E 99, 022137 (2019).

[59] C. De Bacco, A. Guggiola, R. Kühn, and P. Paga, Rare events statistics of random walks on networks: Localisation and other dynamical phase transitions, J. Phys. A 49, 184003 (2016).

[60] S. Attal, F. Petruccione, and I. Sinayskiy, Open quantum walks on graphs, Phys. Lett. A 376, 1545 (2012).

[61] S. Garnerone, Thermodynamic formalism for dissipative quantum walks, Phys. Rev. A 86, 032342 (2012).

[62] K. Macieszczak, M. Guţă, I. Lesanovsky, and J. P. Garrahan, Towards a Theory of Metastability in Open Quantum Dynamics, Phys. Rev. Lett. 116, 240404 (2016).

[63] P. W. Anderson, Absence of diffusion in certain random lattices, Phys. Rev. 109, 1492 (1958).

[64] R. Nandkishore and D. A. Huse, Many-body localization and thermalization in quantum statistical mechanics, Annu. Rev. Condens. Matter Phys. 6, 15 (2015).

[65] F. Iannone, F. Ambrosino, G. Bracco, M. De Rosa, A. Funel, G. Guarnieri, S. Migliori, F. Palombi, G. Ponti, G. Santomauro, and P. Procacci, CRESCO ENEA HPC clusters: A working example of a multifabric GPFS Spectrum Scale layout, in 2019 International Conference on High Performance Computing Simulation (HPCS) (IEEE, Piscataway, New Jersey, 2019), p. 1051. 